\title{
THE LINE SPECTRUM OF THE SOLAR CORONA
}

\author{
P. Swings \\ Pasadena, California
}

For seventy years the solar corona has been observed at practically all eclipse expeditions. It consists of an approximately spherical halo, with faint streamers roughly radial in direction, extending a solar diameter or two from the photosphere, mainly from the spot zones. Curved rays also extend from the polar regions of the sun. The extent and shape of the corona vary appreciably from eclipse to eclipse, being closely related to sunspot activity. Total solar eclipses have provided a wealth of observational material on the corona despite the limited time of totality, seven and a half minutes at most.

Since 1930, the corona has also been studied extensively without an eclipse, using coronagraphs, especially at $\mathrm{Pic}$ du Midi, France, by B. Lyot, ${ }^{1}$ who successfully built the first coronagraph; at Arosa, Switzerland, by M. Waldmeier who has obtained very important observational and theoretical results; and, more recently, at the Harvard station at Climax, Colorado, by W. O. Roberts. Promising experiments by A. M. Skellett and by $\mathrm{H}$. W. Babcock have also been made with a coronaviser designed to observe the corona in full sunlight by photoelectric scanning of an optical image. Eclipse observations of the corona may still be needed, especially for the detection and study of very faint emission lines in the ultraviolet. A number of expeditions are being planned to observe the total solar eclipse of July 9 , 1945. Twenty-two missions of approximately 170 astronomers are being organized in Russia. The longest duration of totality in Russia will be only slightly more than one minute. Other scientific expeditions are being organized by the Swedish and Finnish astronomers for the same eclipse.

Observations with the coronagraph have the obvious advantage of not being strictly limited in time; thus they add much valuable information to the observational data gathered by eclipse

${ }^{1}$ C.R., 202, 1259, 1936; ibid., 203, 1327, 1936; L'Astronomie, 51, 203, 1937 ; C.R., 206, 648, 1938 ; M.N., 99, 580, 1939. 
expeditions. For example, fairly rapid variations in the corona can be studied in detail. It is true that the brightness of the sky must be extremely low (less than $10 \times 10^{-6}$ of the sun) in order that the features of the innermost corona may become observable with a coronagraph. This, according to Waldmeier, happens for only five hours in one hundred winter days at the Arosa Observatory, but "the most ardent eclipse observer can have on the average only one minute per year to secure his observation" (S. A. Mitchell).

The spectrum of the inner corona (extending outward from the limb to a distance of about one-third the diameter of the sun) is mainly continuous, with a number of superimposed emission lines; the solar absorption lines are not visible. In the middle and outer corona, the Fraunhofer absorption lines reappear. The bright-line spectrum is unpolarized, but the continuous radiation shows strong radial polarization. The total radiation from the corona is from about a quarter to a half that of.the full moon, but varies considerably from one eclipse to another.

The bright lines found in the spectrum of the corona are definitely not present in the spectrum of the chromosphere. Just as the nebular lines had first been attributed to an unknown element, "nebulium," the coronal lines were, at first, considered as belonging to a hypothetical element, "coronium." More than twenty lines are present in the inner corona, some of them quite conspicuous, especially a green line $(\lambda 5303)$, two red ones $(\lambda 6375$ and $\lambda 6702)$, three in the infrared $(\lambda \lambda 7892 ; 10,747$; and $10,798)$, and one in the ultraviolet ( $\lambda$ 3388). In most regions of the corona, the green line is the strongest. The wave lengths and contours of the coronal lines have been measured very often. They are not so sharp as the nebular lines but are rather diffuse. Their behavior has been examined at different eclipses; monochromatic images of the corona in various wave lengths have been compared; it has been found that the decrease in intensity of the lines with increasing distance from the limb is not identical for all lines; and certain coronal lines have been observed in the recurring novae RS Ophiuchi, in 1932 and 1942, and T Pyxidis, in 1945. From all these observations the coronal lines have been classified in a number of groups, probably belonging to specific 
excitations. The most reliable of such groupings was made by Lyot. $^{1}$

Yet despite the considerable amount of data accumulated on the coronal lines, no satisfactory identification had been made until 1941. In 1927, after Bowen had succeeded in interpreting the strongest nebular radiations, the coronal lines remained one of the most outstanding spectroscopic mysteries in astronomy.

As previously mentioned, the observations revealed certain groups of lines, each group behaving differently from the others, hence indicating that the coronal lines were certainly not all due to a given atom (or molecule) excited by a specific mechanism. Rather, the lines must belong either to several atoms (or molecules), or to the same atom (or molecule) in different ionization stages, or to the same atom (or molecule) excited by different mechanisms.

A great many attempts to identify the coronal lines have been made since 1911, all of them without success. These attempts will not be reviewed here. ${ }^{2}$ I shall just mention in chronological order a few of the tentative identifications that have been published: hypothetical element (1911), doubly ionized calcium (1922), argon (1929), atomic Raman effect (1930), doubly excited helium (1931, 1934, and 1935), oxygen (1931 to 1933), hydrogen molecule (1932), negative ions (1934), forbidden lines of $N$ II (1936), forbidden lines of $F e$ II (1938).

With the Swedish physicist, B. Edlén, and some of my students in Belgium, I also spent many months between 1932 and 1939 trying vainly all possible mechanisms, including the most unlikely, both for atoms and molecules. Indeed, the situation was very confusing. We were led to believe that the coronal lines must be due to forbidden transitions, since we have the right to think that most permitted lines of all cosmically abundant atoms have been observed in the laboratory. But forbidden transitions always give sharp lines unless the Doppler broadening is large. To produce the observed width of the lines, a temperature of the order of a million degrees is required, which in the past seemed unacceptable for the corona. Moreover, would not the metastable

2 For such a review, see, for example, P. Swings, Scientia, 33, 69, 1939. 
states be depopulated by absorption of solar radiation long before there is a chance for a forbidden downward transition? A basic assumption always made was that atoms or molecules in the corona could be only neutral or ionized not higher than the third stage. We could not imagine a very high ionization in the corona, but this was our fundamental error, as was later evident. In fact, around 1936, Edlén and I reached the conclusion that the last serious possibility of identifying certain coronal lines was with doubly ionized iron. And this is one of the main reasons why we worked for three years on the term-classification of the $F e$ III spectrum; while this work proved useful in astronomy, it did not help any as far as the corona is concerned, except probably in convincing Edlén that the usual assumption of low ionization in the corona had to be dropped.

In 1939, W. Grotrian ${ }^{3}$ pointed out that the term separations $3 \mathrm{~s}^{2} 3 \mathrm{p}^{5}\left({ }^{2} \mathrm{P}_{1 / 2}-{ }^{2} \mathrm{P}_{3 / 2}\right)$ of $F e \mathrm{x}$ and $3 \mathrm{~s}^{2} 3 \mathrm{p}^{4}\left({ }^{3} \mathrm{P}_{1}-{ }^{3} \mathrm{P}_{2}\right)$ of $F e \mathrm{xI}$, as determined in the extreme ultraviolet by Edlén, ${ }^{5}$ coincide with the wave numbers of the two coronal lines $\lambda 6374$ and $\lambda$ 7892. This remark by Grotrian came at about the time Edlén was preparing his report on the spectra of novae in their nebular stages which was to be presented at the Paris conference on novae. Edlén became deeply interested in Grotrian's remark. From Edlén's unpublished measurements of the spectra of $C a$ XII and $C a$ XIII he found that the separations in the $2 \mathrm{~s}^{2} 2 \mathrm{p}^{5}$ and $2 \mathrm{~s}^{2} 2 \mathrm{p}^{4}$ configurations of these two ions also coincided, within the experimental errors, with two fainter coronal lines, $\lambda 3328$ and $\lambda$ 4086. Assuming the identifications of $F e x$ and XI and $C a$ XII and XIII to be correct, Edlén proceeded with the prediction of the forbidden lines of $\mathrm{Fe}$ xIII and xIV, $\mathrm{Ni}$ xII, etc., and found a number of coincidences which even at that time (June-July, 1939) seemed too remarkable to be due purely to coincidence. I had the privilege of seeing this first table and of discussing it with Edlén in

3 Naturwissenschaften, 27, 214, 1939.

4 Throughout this paper the levels are named in the order: initial (upper) level minus end (lower) level. This is not the customary convention but it conforms to the notations used by Edlén.

${ }^{5}$ Zs. f. Phys., 103, 536, 1936; ibid., 104, 188 and 407, 1937. 
June-July, 1939, while he was spending a few weeks in my laboratory in Belgium on his way to and from the Paris meeting. Yet he refrained from mentioning his results at the conference even when he was questioned on the problem of coronal lines in novae: modestly, he wanted to study the matter further. His first announcement in a scientific periodical appeared in 1941 under the title "An Attempt to Identify the Emission Lines in the Spectrum of the Solar Corona."' A complete account was later published in the Zeitschrift für Astrophysik, 22, 30, 1942.

It may be convenient to divide the coronal problem into two parts: (1) the problem of coronium, comprising the identification of the lines and the determination of the ionization and excitation conditions necessary to produce these lines; (2) the problem of the corona, consisting of the physical explanation of the presence of the identified atoms and of their ionization and excitation. Evidently these two problems are strongly interdependent. But while the problem of coronium has now been solved satisfactorily, the problem of the corona still remains very mysterious. This present paper will deal essentially with the problem of coronium.

A few recent observational results obtained by Lyot and by Waldmeier should be mentioned here. Using a polarizing monochromatic filter which isolates spectral bands $2 \mathrm{~A}$ wide at various wave lengths, Lyot obtained simultaneous motion pictures of the corona in the red line, the corona in the green line, and the prominences in $H \alpha$. These films, some of which were continued for more than twelve hours, cover a total duration of 92 hours. Even when speeded 2400 times, the corona remains stationary, which is at variance with the often repeated statement that there are rapid motions in the corona. "The arches and jets appear and fade progressively along invisible trajectories, and the corona thus modifies its shape and aspect without perceptible motions" (Lyot). On the other hand, the films confirm the difference between the distribution of the red and the green lines in the corona.

Waldmeier's numerous papers published during the war also

${ }^{6}$ B. Edlén, Arkiv f. Matem., Astr. och Fysik, 28, B, No. 1, 1941. For a detailed review of Edlén's papers, see P. Swings, Ap. J., 98, 116, 1943. 
form an important contribution to our knowledge of the corona. ${ }^{7}$ The average intensity distribution of $\lambda 5303$ with heliocentric latitude shows an equatorial minimum (say intensity 10), a primary maximum in the spot zones (intensity 20), a secondary maximum near $\pm 60^{\circ}$ (intensity 4 ), and a minimum (intensity smaller than 1) at the poles. By comparison of his observations with maps of the photosphere, Waldmeier finds that coronal emission is generally most intense over the spot zones, each individual spot underlying a region of maximum coronal activity. However, bright coronal regions may also lie over unspotted areas, as for example in the latitude region $\pm 60^{\circ}$. There may be "green regions" where the green line is abnormally strong and "red regions" where the red line is especially intense. Lines that are usually weak may occasionally reach considerable intensities; for example, $\lambda$ 5694, which normally has an intensity less than 5 per cent of that of the green line, was once found to be three times as strong as $\lambda 5303$ over a very large active spot group surmounted by prominences.

Waldmeier calls "C-region" a region in which the green line is abnormally strong. It seems that the C-regions are related to terrestrial magnetic storms and auroras which often occur 7.4 days after a C-region is observed at the east limb or 6.2 days before one is at the west limb. Since one-quarter of the solar period is 6.8 days, these observations would indicate a transit time of 0.6 day or a mean velocity of $3000 \mathrm{~km} / \mathrm{sec}$ for the corpuscular emission responsible for the magnetic storms. From Waldmeier's observations, one is indeed tempted to identify the $\mathrm{C}$-regions with the solar areas responsible for the magnetic disturbances. Evidently this point deserves and requires additional discussion.

THE PROBLEM OF CORONIUM

Wave-number coincidences with directly observed term separations.-The coincidences between the level separations of $F e \mathrm{x}$ and XI and $C a$ XII and XIII as determined in the laboratory by

${ }^{7}$ For a detailed review of these papers, see A. J. Deutsch, $A p . J ., 101$, $117,1945$. 
Edlén ${ }^{5}$ and the wave numbers of coronal lines are listed in Table I.

TABLE I

The Experimental Basis for the Direct Identífication of Coronal Lines

\begin{tabular}{|c|c|c|c|c|}
\hline \multicolumn{3}{|c|}{ Laboratory Data } & \multicolumn{2}{|c|}{ Corona } \\
\hline $\begin{array}{c}\text { Electron } \\
\text { Configurations }\end{array}$ & $\begin{array}{l}\text { Forbidden } \\
\text { Transition }\end{array}$ & $\begin{array}{c}\text { Separation as } \\
\text { Obtained from } \\
\text { Observed } \\
\text { Laboratory } \\
\text { Lines }\end{array}$ & $\begin{array}{l}\text { Wave } \\
\text { Number }\end{array}$ & $\begin{array}{l}\text { Wave } \\
\text { Length }\end{array}$ \\
\hline$F e \times 3 \mathrm{~s}^{2} 3 \mathrm{p}^{5}$ & ${ }^{2} \mathrm{P}_{1 / 2}-{ }^{2} \mathrm{P}_{3 / 2}$ & $15,687 \mathrm{~cm}^{-1}$ & $15,683 \mathrm{~cm}^{-1}$ & $6374.5 \mathrm{~A}$ \\
\hline$F e \times I 3 \mathrm{~s}^{2} 3 \mathrm{p}^{4}$ & ${ }^{3} \mathrm{P}_{1}-{ }^{3} \mathrm{P}_{2}$ & 12,673 & 12,668 & 7891.9 \\
\hline$C a \times I I 2 s^{2} 2 p^{5}$ & ${ }^{2} \mathrm{P}_{1 / 2}-{ }^{2} \mathrm{P}_{3 / 2}$ & 30,028 & 30,039 & 3328 \\
\hline$C a \times \operatorname{xII} 2 \mathrm{~s}^{2} 2 \mathrm{p}^{4}$ & ${ }^{3} \mathrm{P}_{1}-{ }^{3} \mathrm{P}_{2}$ & 24,464 & 24,465 & 4086.3 \\
\hline
\end{tabular}

The separations listed in column 3 of Table I were obtained from laboratory lines in the region around $90 \mathrm{~A}$ for $F e \mathrm{x}$ and XI and around $150 \mathrm{~A}$ for $\mathrm{Ca}$ XII and xirr. The wave-number values of these separations are therefore uncertain and, although the coincidences of Table I are impressive, they cannot quite make the identification conclusive, owing to the unavoidable relatively wide tolerances. The decisive fact is that, assuming the identifications of $\lambda \lambda 6374(F e \mathrm{x}), 7892$ ( $F e \mathrm{xI}$ ), 3328 ( $\mathrm{Ca}$ xII), and 4086 ( $C a$ XIIr) to be correct, an almost complete identification of the coronal spectrum can be consistently carried through, as will now be shown. First, it should be noticed that the [Fe vII] lines which have been observed in several novae and peculiar stars do not appear in the corona. Besides, the ground configuration of $F e \mathrm{IX}$ gives only one single level, and $F e \mathrm{Ix}$ has no metastable state. As for $F e$ virr, the splitting of the ground term ${ }^{2} \mathrm{D}$ is too small to give any astronomically observable transition. Hence, as far as iron is concerned, the investigation should be concerned with $F e \mathrm{x}$ and higher stages of ionization. Similar considerations apply to other atoms, especially nickel. A survey of possible level separations shows that coronal transitions should be searched for mainly within the configurations $3 \mathrm{~s}^{2} 3 \mathrm{p}, 3 \mathrm{~s}^{2} 3 \mathrm{p}^{2}$, $3 \mathrm{~s}^{2} 3 \mathrm{p}^{4}$, and $3 \mathrm{~s}^{2} 3 \mathrm{p}^{5}$ of the elements in the iron group. The $3 \mathrm{~s}^{2} 3 \mathrm{p}^{3}$. 
configuration ( $\mathrm{Fe}$ XII and $\mathrm{Ni}$ XIV) cannot produce forbidden lines of appreciable intensity, as will be shown later. Because of the relative cosmical abundances, iron should be expected first, nickel next.

Term intervals in the configurations $3 s^{2} 3 p, 3 s^{2} 3 p^{2}, 3 s^{2} 3 p^{4}$, and $3 s^{2} 3 p^{5}$ of $\mathrm{Fe}$ and $\mathrm{Ni}$.- The isoelectronic sequences considered here are:

$3 \mathrm{~s}^{2} 3 \mathrm{p}: A l$ г $, S i \mathrm{II}, \ldots \ldots, S c \mathrm{Ix}, \ldots . ., F e$ xrv, $C o \mathrm{xv}, N i \mathrm{xvI}$ (atomic number $\mathrm{Z}$ from 13 to 28 );

$3 \mathrm{~s}^{2} 3 \mathrm{p}^{5}: C l$ ч,$A$ II, $\ldots \ldots, V$ vir, $\ldots . ., F e$ x, Co xI, Ni xir ; $3 \mathrm{~s}^{2} 3 \mathrm{p}^{2}: S i$ I, $P$ II, $\ldots . ., F e \times$ xirl, $C o x \mathrm{xIv}, N i \mathrm{xv}$; $3 \mathrm{~s}^{2} 3 \mathrm{p}^{4}: S \mathrm{I}, C l$ II, $. . ., F, F e$ xi, Co xir, $N i$ xirr.

The configurations $3 \mathrm{~s}^{2} 3 \mathrm{p}$ and $3 \mathrm{~s}^{2} 3 \mathrm{p}^{5}$ give rise to a ${ }^{2} \mathrm{P}$ term only, whose splitting accurately follows the regular doublet law; in other words, the wave-number value of the ${ }^{2} \mathrm{P}$ splitting varies with the atomic number $Z$ as $(Z-\sigma)^{4}$, the screening factor $\sigma$ being nearly constant. Accurate laboratory values of the splitting have been obtained up to $S c$ Ix in the $3 \mathrm{~s}^{2} 3 \mathrm{p}$ sequence and up to $V$ VII in the $3 \mathrm{~s}^{2} 3 \mathrm{p}^{5}$ sequence. ${ }^{8}$ Extrapolations revealed at once the identifications schematized in Figure 1 ( $a$ and $b)$; the $[F e$ XIV] transition gives in fact the strong green line. It will be shown later that the intensity ratios of homologous lines of iron and nickel are in accordance with the relative cosmical abundance of these elements, simultaneously indicating the degree of ionization of the coronal matter.

The extrapolation problem is more complex for the configurations $3 \mathrm{~s}^{2} 3 \mathrm{p}^{2}$ and $3 \mathrm{~s}^{2} 3 \mathrm{p}^{4}$, which, in order of increasing energy, furnish respectively the following levels: ${ }^{3} \mathrm{P}_{0},{ }^{3} \mathrm{P}_{1},{ }^{3} \mathrm{P}_{2},{ }^{1} \mathrm{D}_{2},{ }^{1} \mathrm{~S}_{0}$, and ${ }^{3} \mathrm{P}_{2},{ }^{3} \mathrm{P}_{1},{ }^{3} \mathrm{P}_{0},{ }^{1} \mathrm{D}_{2},{ }^{1} \mathrm{~S}_{0}$. The ${ }^{1} \mathrm{~S}_{0}$ level lies too high above ${ }^{1} \mathrm{D}_{2}$ to give observable lines. But, besides the transitions between the ${ }^{3} \mathrm{P}$ components which are analogous to those of the $3 \mathrm{~s}^{2} 3 \mathrm{p}$ or $3 \mathrm{~s}^{2} 3 \mathrm{p}^{5}$ configurations, we have also to consider the transitions from ${ }^{1} \mathrm{D}_{2}$ to ${ }^{3} \mathrm{P}$. It will be apparent from what follows that coronal lines might be expected especially for the following

${ }^{8}$ In the $3 \mathrm{~s}^{2} 3 \mathrm{p}^{5}$ sequence, laboratory values of the splitting have been obtained also from $C r$ viII to $C o x I$, but they are of lower precision. 
transitions: ${ }^{1} \mathrm{D}_{2}-{ }^{3} \mathrm{P}_{2},{ }^{3} \mathrm{P}_{2}-{ }^{3} \mathrm{P}_{1},{ }^{3} \mathrm{P}_{1}-{ }^{3} \mathrm{P}_{0}$ of $3 \mathrm{~s}^{2} 3 \mathrm{p}^{2}$, and ${ }^{1} \mathrm{D}_{2}-{ }^{3} \mathrm{P}_{1}$, ${ }^{3} \mathrm{P}_{1}-{ }^{3} \mathrm{P}_{2}$ of $3 \mathrm{~s}^{2} 3 \mathrm{p}^{4}$. Moreover, the extrapolation problem is rendered difficult in this case because of the progressive change from LS to JJ coupling when $Z$ increases along an isoelectronic sequence, requiring thus the use of the more complicated theoretical formulae for intermediate coupling. Yet the results of the extrapolation are of an accuracy similar to that for $3 \mathrm{~s}^{2} 3 \mathrm{p}$ and $3 \mathrm{~s}^{2} 3 \mathrm{p}^{5}$. The results are schematized in Figure $1(c$ and $d)$. The only difficulty concerns $F e$ xIII, for which it is impossible to de-

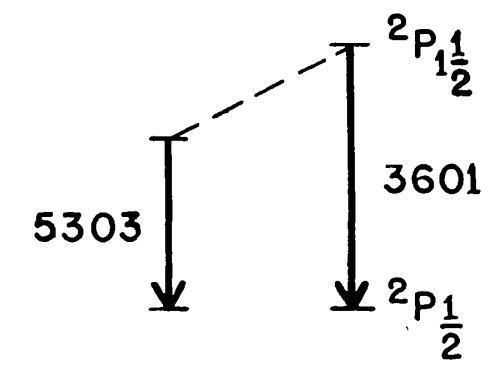

Fe XIV NiXVI

(a)

$$
3 s^{2} 3 p
$$

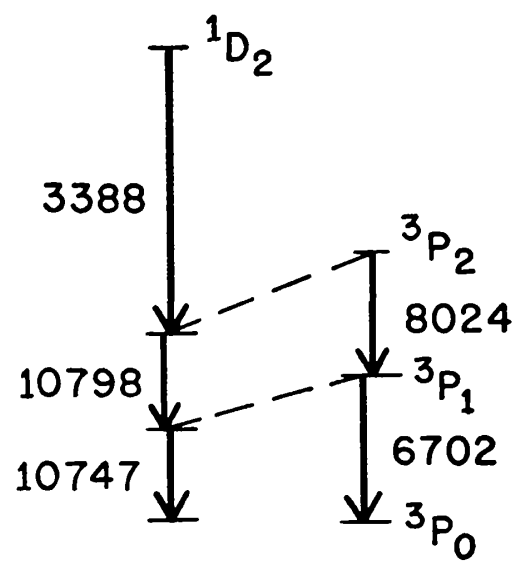

Fe XIII Ni XV

(c)

$$
3 s^{2} 3 p^{2}
$$

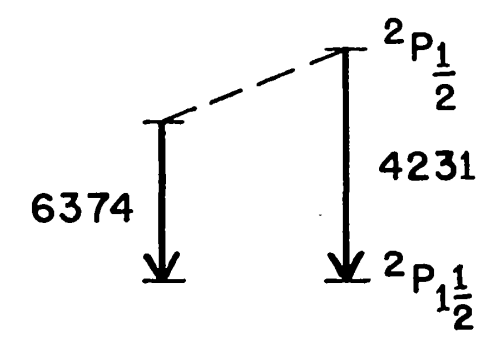

FeX NiXII

(b) $3 s^{2} 3 p^{5}$

FIG. 1.- $-3 \mathrm{~s}^{2} 3 \mathrm{p}^{\mathrm{k}}$ configurations of iron and nickel ions and the corresponding coronal lines. 
cide, from the extrapolated wave numbers alone, how to allot the two very close infrared coronal lines to ${ }^{3} \mathrm{P}_{1}-{ }^{3} \mathrm{P}_{0}$ or to ${ }^{3} \mathrm{P}_{2}-{ }^{3} \mathrm{P}_{1}$. However, a definite identification will be possible on the basis of line intensities. The ${ }^{1} \mathrm{D}_{2}-{ }^{3} \mathrm{P}_{2}$ transition of $N i \mathrm{xV}$ falls outside the observable spectral range. All in all, at the present stage, 13 coincidences have been observed between the wave numbers of coronal lines and those of transitions in the ground configurations $3 \mathrm{~s}^{2} 3 \mathrm{p}, 3 \mathrm{~s}^{2} 3 \mathrm{p}^{2}, 3 \mathrm{~s}^{2} 3 \mathrm{p}^{4}$, and $3 \mathrm{~s}^{2} 3 \mathrm{p}^{5}$ of $F e$ and $N i$. These 13 coronal lines account for more than nine-tenths of the total intensity of the coronal-line emission.

The next step is a discussion of the observed intensities in order to ascertain whether these are compatible with the theoretical transition probabilities, with the cosmical abundances of the elements, and with definable ionization and excitation conditions in the corona.

Line intensities.-There is no initial state of the ions considered for which more than one transition has been identified in the corona. Hence the intensity ratios of the various forbidden lines depend upon the physical conditions. In this connection it should be remembered that marked variations in the intensity ratios of the coronal lines have been repeatedly observed, as already mentioned in the beginning.

The intensity of a forbidden transition of probability $A_{1}$ and wave number $v$ may be expressed in arbitrary units by the classical formula

$$
I=\frac{n v A_{1}}{A_{1}+A_{2}+A_{3}+\ldots+B+C}
$$

where $n$ is the number of ions arriving in the initial metastable state per second; $A_{1}, A_{2}, A_{3}$ are the probabilities of the various spontaneous transitions from the initial state; and $B$ and $C$ are the probabilities of de-excitation by collision and radiative absorption.

It is possible to determine fairly accurately the probabilities $A$ of the transitions considered here. For all lines considered in the corona the probabilities of the electric quadrupole are extremely small compared with the probabilities of the magnetic dipole. Edlén based his determination of the $A$ values on recent 
investigations by S. Pasternack ${ }^{9}$ and by Shortley, Aller, Baker, and Menzel. ${ }^{10}$ A general result of interest is that the transition probabilities $A$ of the strongest coronal lines are much greater than those of the forbidden lines observed in nebulae.

De-excitation by absorption of radiation is probably unimportant, since the next higher configuration, $3 \mathrm{~s} 3 \mathrm{p}^{\mathrm{k}+1}$, combining with the ground configuration, $3 \mathrm{~s}^{2} 3 \mathrm{p}^{\mathrm{k}}$, corresponds to an excitation potential of about 35 volts in all the $F e$ ions and somewhat more in the $N i$ ions. In any case, the de-exciting radiation must be of wave length shorter than $\lambda 400$, and the intensity of the solar radiation in this spectral region is probably extremely small.

Incidentally this takes care of one of the objections always raised against the hypothesis that the coronal lines may be due to forbidden transitions : the objection is not valid for highly ionized atoms.

Edlén gives convincing reasons why the de-excitation of the metastable levels by electron collisions $\left({ }^{3} \mathrm{P}_{0}\right.$ excluded) can be neglected in comparison with the spontaneous transitions. This conclusion can even be reached without any calculation, in the following way. If de-excitations by electron collisions were much in excess of those by radiative emission, a Boltzmann distribution would result; and, at a high electron temperature ( $T_{e} \approx 250,000^{\circ}$ or more), the number of ions in the various levels of the ground configuration would be approximately proportional to their statistical weights. Consequently the line intensities for a given ion would be proportional to $(2 J+1) A v$. In $\mathrm{Fe}$ xIII, for example, the intensity ratios of the transition ${ }^{1} \mathrm{D}_{2}-{ }^{3} \mathrm{P}_{2},{ }^{3} \mathrm{P}_{2}-{ }^{3} \mathrm{P}_{1},{ }^{3} \mathrm{P}_{1}-{ }^{3} \mathrm{P}_{0}$ would be given approximately as $128: 4.6: 3.9$, while the observed intensity ratios for the corresponding coronal lines $\lambda \lambda 3388,10,798$, and 10,747 are $16: 35: 55$, according to Lyot. ${ }^{11}$ Such a discrepancy is too large to be attributed to observational uncertainties. In comparing the probabilities $A$ and $B$, the fact that the electron density in the corona

9 Ap.J., 92, 129, 1940.

10 Ap. J., 93, 178, 1941.

11 C.R., 202, 1259, 1936 ; ibid., 203, 1327, 1936 ; L'Astronomie, 51, 203, 1937 ; C.R., 206, 648, 1938; M.N., 99, 580, 1939. 
$\left(N_{e}[\right.$ cor. $] \approx 10^{8} \mathrm{~cm}^{-3}$, according to Baumbach $\left.{ }^{12}\right)$ is lighter than in most nebulae $\left(N_{e}[\right.$ neb. $\left.] \approx 10^{4} \mathrm{~cm}^{-3}\right)$ is compensated by the much greater transition probabilities of the coronal transitions and also, to a lesser extent, by the higher electron temperature of the corona.

In order to be able to apply formula (1) the only remaining factor to be determined is the number of excitations per cubic centimeter and per second into certain levels. Edlén computes these numbers for excitation by collisions of electrons having a Maxwellian velocity distribution corresponding to the temperature $T_{e}$. But he mentions the fact that the conditions for excitation by radiation are considerably more favorable in the corona than they are in nebulae. In the first place, the ratio between exciting and excited intensity is of a much higher order of magnitude; and, in the second place, the corresponding transition probabilities are roughly $10^{4}$ times greater. No attempt is being made to estimate the amount of an eventual radiative excitation.

I shall not go into the details of Edlén's calculations. The essential results are:

1. When several transitions are observed in the same ion, as they are for $\mathrm{Fe}$ xI, $\mathrm{Fe}$ xIII, and $\mathrm{Ni} \mathrm{xv}$, the observed relative intensities of the coronal lines agree satisfactorily with the computed intensities if the adopted electron temperature is of the order of $250,000^{\circ}$. From the extrapolated term separations alone, it was impossible to decide how each one of the two close infrared lines $\lambda 10,747$ and $\lambda 10,798$ is to be identified with the separate transitions ${ }^{3} \mathrm{P}_{1}-{ }^{3} \mathrm{P}_{0}$ and ${ }^{3} \mathrm{P}_{2}-{ }^{3} \mathrm{P}_{1}$. The assignment was primarily made to correspond with the intensity ratio of the analogous $N i \mathrm{xv}$ lines. This is now confirmed by the calculated intensities.

2. The observed relative intensities of lines attributed to different ionization stages indicate an ionization maximum in stages XIII or XIV. About half of the coronal atoms are probably in the stages XIII and Xrv, and probably more than 95 per cent of the atoms are in the stages $\mathrm{x}$ to $\mathrm{xVI}$.

3. The relative intensities of the lines of iron and nickel are

12 A.N., 263, 121, 1937. 
compatible with the average abundance ratio found in meteorites $^{13}$ or estimated in cosmical sources.

Other ionization stages of iron and nickel.-From the foregoing considerations it is clear that transitions between levels of the ground configurations of $F e \mathrm{x}, \mathrm{xI}, \mathrm{xIII}, \mathrm{xIV}$, and of $N i \mathrm{xII}$, $\mathrm{xIII}, \mathrm{xV}$, XVI produce most of the coronal lines. Although $F e$ XII and $N i$ xIV must be abundant in the corona, we should not expect any coronal lines to be attributed to them because of the relative positions of the levels of their $3 \mathrm{~s}^{2} 3 \mathrm{p}^{3}$ configuration. The term ${ }^{2} \mathrm{D}$ is too high, relative to the ground level ${ }^{4} \mathrm{~S}$; and the splittings of ${ }^{2} \mathrm{D}$ and ${ }^{2} \mathrm{P}$ are too small to produce observable lines. Only ${ }^{2} \mathrm{P}_{1 / 2}-^{2} \mathrm{D}_{5 / 2}$ could fall within the observable range, but this transition has a vanishingly low probability (Fig. 2). The lower

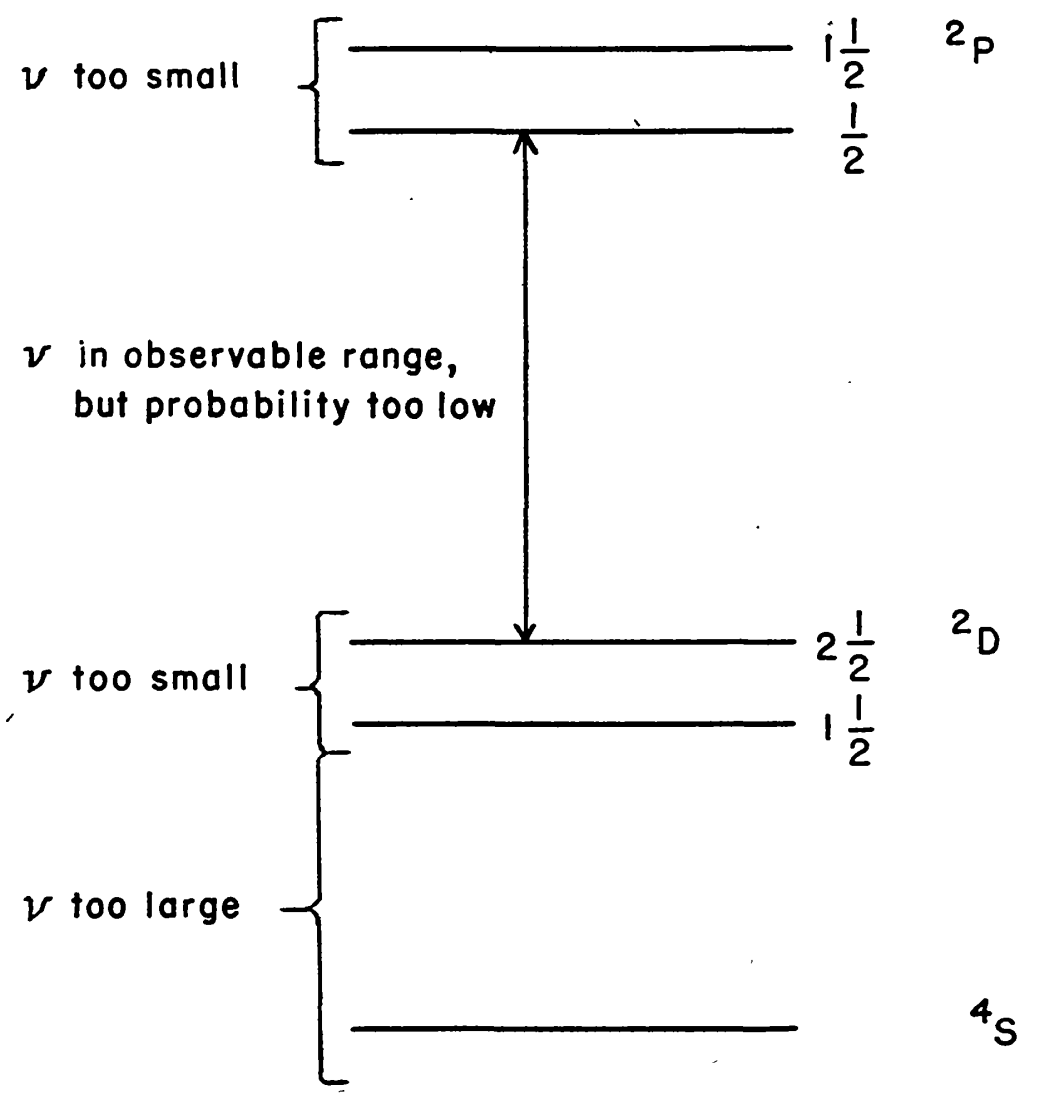

FIG. $2 .-3 \mathrm{~s}^{2} 3 \mathrm{p}^{3}$ configuration of $F e$ XII and $N i$ XIV.

13 V. M. Goldschmidt, Norske Videnskaps Akademie Skrifter. I. Mat. Naturv. Klasse, No. 4, 1938. 
stages of ionization have already been considered. Their absence is in accordance with the degree of ionization already estimated for the corona. The ground configurations of $F e \mathrm{xV}, \mathrm{xVI}$, and $\mathrm{xVII}$ and the corresponding $N i$ ions give only one single level ${ }^{1} \mathrm{~S}_{0}$ or ${ }^{2} \mathrm{~S}_{1 / 2}$. Stages higher than $F e$ xvII are improbable, since the ionization increases abruptly in passing from $F e$ XVII to $F e$ XvirI when the $\mathrm{L}$ shell is broken up.

Although no further transition within the ground configuration of $\mathrm{Fe}$ and $\mathrm{Ni}$ ions can therefore be expected, we may still consider the second lowest configuration of $F e \mathrm{xV}$ and $N i \mathrm{x} \cdot \mathrm{VII}$ in which two metastable levels occur, namely, $3 \mathrm{~s} 3 \mathrm{p}\left({ }^{3} \mathrm{P}_{0}\right)$ and $\left({ }^{3} \mathrm{P}_{2}\right)$. These levels cannot combine with the ground level $3 \mathrm{~s}^{2}\left({ }^{1} \mathrm{~S}_{0}\right)$ under any circumstances, but the transition between ${ }^{3} \mathrm{P}_{2}$ and ${ }^{3} \mathrm{P}_{1}$ is possible through magnetic dipole radiation. From Edlén's study of the ultraviolet spectrum of $F e \mathrm{xv}$ the wave number of the $3 \mathrm{~s} 3 \mathrm{p}\left({ }^{3} \mathrm{P}_{2}-{ }^{3} \mathrm{P}_{1}\right)$ transition may be estimated as $14,120 \mathrm{~cm}^{-1}$, which is close enough to be identified with the only remaining infrared line $\lambda 7059\left(v=14,161 \mathrm{~cm}^{-1}\right)$. This identification is very probable. The excitation energy, 31.9 volts, is considerably higher than for the previously identified transitions; but this does not constitute any objection if the electron temperature is of the order of from $200,000^{\circ}$ to $400,000^{\circ}$.

Nine metastable states with excitation energies of the order of 50 volts also occur in $F_{i}$ IX and $N i$ xI. They belong to the $3 \mathrm{~s}^{2} 3 \mathrm{p}^{5} 3 \mathrm{~d}$ configuration; and a number of combinations may arise between them, some of the lines falling in the observable region. Their wave lengths cannot be predicted at present. Yet it is not impossible that some faint coronal lines may be explained as such transitions in $F e$ Ix and $N i$ xI.

Forbidden lines of other highly ionized atoms.-Considering the great intensity of certain transitions of $\mathrm{Fe}$ and $\mathrm{Ni}$, it seems reasonable to search for the corresponding transitions of the neighboring atoms with the configuration $3 \mathrm{~s}^{2} 3 \mathrm{p}^{\mathrm{k}}$. The wave numbers of the transitions appearing in the observable region can be accurately predicted for $C o x v, M n x I I I, S x I I, C r$ xII, and $\mathrm{Co}$ xIV with the aid of the $\mathrm{Fe}$ and $\mathrm{Ni}$ identifications, but no coincidence with a coronal line appears. This may readily be explained, however, if the chemical composition of the corona is 
- similar to that of the solar atmosphere or to that of meteorites. Weak coronal lines may eventually be found at the wave lengths listed in Table II, which correspond to such transitions.

\section{TABLE II}

Estimated Wave Lengths of Some Transitions Not Yet Observed

\begin{tabular}{c|l|c|c}
\hline \hline Transition & $\lambda$ Air & Transition & $\lambda$ Air \\
\cline { 2 - 4 }${ }^{2} \mathrm{P}_{3 / 2}-{ }^{2} \mathrm{P}_{1 / 2}$ & 4349.4 & $\operatorname{Cr} \times \operatorname{CII~}^{2} \mathrm{P}_{3 / 2}-{ }^{2} \mathrm{P}_{1 / 2}$ & 8159 \\
$M n \times I I I{ }^{2} \mathrm{P}_{3 / 2}-{ }^{2} \mathrm{P}_{1 / 2}$ & 6539 & $\operatorname{CoxIV} \mathrm{P}_{1}-{ }^{3} \mathrm{P}_{0}$ & 8448 \\
$S \times I I{ }^{2} \mathrm{P}_{3 / 2}-{ }^{2} \mathrm{P}_{1 / 2}$ & 7536 & & \\
\hline
\end{tabular}

Considering the fact that two coronal lines have been satisfactorily attributed to forbidden transitions in $C a$ xIII $\left(2 \mathrm{~s}^{2} 2 \mathrm{p}^{4}\right)$ and $C a$ XII $\left(2 \mathrm{~s}^{2} 2 \mathrm{p}^{5}\right)$, we should also examine the other possible transitions within configurations $2 \mathrm{~s}^{2} 2 \mathrm{p}^{\mathrm{k}}$. In the $2 \mathrm{~s}^{2} 2 \mathrm{p}^{5}$ sequence, $A \mathrm{x}$ provides the identification of the coronal line $\lambda 5536$ $\left({ }^{2} \mathrm{P}_{1 / 2}-{ }^{2} \mathrm{P}_{3 / 2}\right)$. In the $2 \mathrm{~s}^{2} 2 \mathrm{p}$ sequence, $A$ xIV probably explains the coronal line $\lambda 4359$, but this is not certain. In the $2 \mathrm{~s}^{2} 2 \mathrm{p}^{2}$ sequence, $C a \mathrm{xv}$ may explain a coronal line found at $\lambda$ 5694, but this is uncertain because the ionization potential of $C a$ XIV is considerably higher than for any other observed coronal ion.

No forbidden or permitted line of Si, $M g, C, N, O$, or $N e$ is possible in the corona under the actual ionization conditions.

Conclusion.-The main results are collected in Table III. The wave lengths of the coronal lines are from Mitchell's compilation $^{14}$ and from Lyot's later measurements. The intensities are from Grotrian (col. 3) and Lyot (reduced values, col. 4). The sixth column gives the transition probability: all the identified lines are due to magnetic dipole radiation. The seventh and eighth columns give the excitation potential and the ionization potential of the next preceding ionization stages. Only six coronal lines $(\lambda \lambda 3454,4567,3801,4311,4412$, and 4586) remain unidentified, the latter four being very faint.

14 Handb. d. Ap., Vols. 4 and 7. 
TABLE III

Emission Lines in the Solar Corona

\begin{tabular}{|c|c|c|c|c|c|c|c|}
\hline$\lambda$ & $v \mathrm{Cm}^{-1}$ & Inte & & Identification & $A_{m} \mathrm{Sec}^{-1 *}$ & $\underset{\substack{\text { E.P. } \\
\text { Volts }}}{\text {. }}$ & $\begin{array}{l}\text { I.P. } \\
\text { Volts }\end{array}$ \\
\hline 3328 & 30039 & 1.0 & $\cdots$ & Ca XII & 488 & 3.72 & 589 \\
\hline 3388.1 & 29507 & 16 & . & $\mathrm{Fe}$ XIII & 87 & 5.96 & 325 \\
\hline 3454.1 & 28943 & 2.3 & .. & & & & \\
\hline 3601.0 & 27762 & 2.1 & .. & $N i \mathrm{XVI}$ & 193 & 3.44 & 455 \\
\hline 3642.9 & 27443 & $\ldots$ & & $N i \mathrm{XIII}$ & 18 & 5.82 & 350 \\
\hline 3800.8 & 26303 & 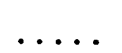 & & & & & \\
\hline 3986.9 & 25075 & 0.7 & $\ldots$ & $\mathrm{Fe} \times \mathrm{XI}$ & 9.5 & 4.68 & 261 \\
\hline 4086.3 & 24465 & 1.0 & $\ldots$ & CaXIII & 319 & 3.03 & 655 \\
\hline 4231.4 & 23626 & 2.6 & $\ldots$ & $N i \mathrm{XII}$ & 237 & 2.93 & 318 \\
\hline 4311 & 23190 & .. & $\cdots$ & 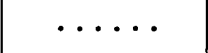 & . & & $\cdots$ \\
\hline 4359 & 22935 & & & $? A \times \mathrm{xIV}$ & 108 & 2.84 & 682 \\
\hline 4412 & 22659 & . & $\cdots$ & … & $\cdots$ & $\cdots$ & $\cdots$ \\
\hline 4567 & 21890 & 1.1 & $\ldots$ & $\cdots$ & . & $\ldots$ & $\cdots$ \\
\hline 4586 & 21799 & & & & & $\cdots$ & \\
\hline 5116.03 & 19541.0 & 4.3 & 2.2 & $N i \mathrm{XIII}$ & 157 & 2.42 & 350 \\
\hline 5302.86 & 18852.5 & 100 & 100 & $\mathrm{Fe} \times \mathrm{XIV}$ & 60 & 2.34 & 355 \\
\hline 5536 & 18059 & $\ldots \ldots$ & & $A \mathrm{x}$ & 106 & 2.24 & 421 \\
\hline 5694.42 & 17556.2 & $\ldots$ & 1.2 & $? C a \times \mathrm{x}$ & 95 & 2.18 & 814 \\
\hline 6374.51 & 15683.2 & 8.1 & 18 & $F e \mathrm{x}$ & 69 & 1.94 & 233 \\
\hline 6701.83 & 14917.2 & 5.4 & 2.0 & $N i \mathrm{xv}$ & 57 & 1.85 & 422 \\
\hline 7059.62 & 14161 & & 2.2 & $F$ & & 31.7 & 390 \\
\hline 7891.94 & 12667.7 & & 13 & $\mathrm{Fe}$ & 44 & 1.57 & 261 \\
\hline 8024.21 & 12458.9 & $\cdots$ & 0.5 & $N i \mathrm{xv}$ & 22 & 3.39 & 422 \\
\hline 10746.80 & 9302.5 & $\cdots$ & 55 & $F e \times I I I$ & 14 & 1.15 & 325 \\
\hline 10797.95 & 9258.5 & $\ldots \ldots$ & 35 & $F e$ XIII & 9.7 & 2.30 & 325 \\
\hline
\end{tabular}

* These are the probabilities computed by Edlén; more recent values determined by Kun Huang $(A p . J ., 101,187,1945)$ do not differ appreciably.

$\dagger$ The ionization potential refers to the next lower ionization stage.

In the light of the present identifications, a physical significance may be attributed to the widths of the coronal lines. As shown by Lyot and by Waldmeier, the line profiles are symmetrical and entirely explainable by the Doppler effect of a Maxwellian velocity distribution. The kinetic temperature derived from the observed widths is of the same order of magnitude as the temperature estimated from the established degree of ioniza- 
tion. The identifications also agree perfectly with the classification of coronal lines in groups which, according to Lyot, appear strengthened or weakened in certain coronal regions.

This brings us to the end of our sketchy discussion of the problem of coronium. A few pages will now be devoted to the problem of the corona.

THE PROBLEM OF THE CORONA

First, let. us see how the high excitation implied by Edlén's identification of the coronal lines fits into the general picture provided by the observations. How is it that the Balmer lines of hydrogen are not observed or are extremely weak compared with [Fe xIv]? Hydrogen is, by far, the most abundant constituent of practically all stellar atmospheres, including the solar atmosphere. It is probably abundant in the corona also, where it must be mostly ionized. The protons should capture electrons and, by recombination, give rise to the hydrogen spectrum. What hampers this mechanism of recombination? The logical answer seems to be that, on account of the very high temperature, the capture process happens rarely. Actually, if this is put in mathematical form, it is found that a temperature of several hundred thousand degrees must exist in the corona: this was shown conclusively by Menzel and Goldberg. ${ }^{15}$ I understand that Lyot has recently observed $H \alpha$ in the solar corona with an intensity less than $1 / 10,000$ that of the green line. The estimated relative intensities of $H \alpha$ and the forbidden lines should help in estimating the electron temperature in the corona.

Another point to consider is that of the intense continuous spectrum of the inner corona. The polarization and color distribution of this continuum indicate that it is due to the scattering of solar radiation by free electrons. How is it that the continuum of the inner corona does not show the solar absorption lines? Even the very strong $\mathrm{H}$ and $\mathrm{K}$ solar lines of $\mathrm{Ca}$ II are hardly visible and considerably broadened in the inner corona. The smoothing out of the absorption lines is probably due to the very swift motions of the scattering electrons. In other. words,

${ }_{15}$ Telescope, 8, 65, 1941. 
the blurring of the Fraunhofer lines would be due to the same cause as the width of the coronal lines. But since electrons are 100,000 times lighter than iron atoms, their velocities, and hence the line broadening caused by these velocities, must be very much larger. The observed broadening would require an electron temperature of the order of 350,000 degrees.

Astronomers had previously been led to think that the excitation conditions were higher in certain regions of the chromosphere than in the reversing layer. Such an assumption of superexcitation was based on the otherwise unexplained observation of emission lines of ionized helium in the flash spectrum.

The distribution of electronic density in the corona has been derived by S. Baumbach, ${ }^{16}$ using all available photometric observations and assuming that the coronal continuum consists of photospheric light scattered by free electrons. H. Alfvén ${ }^{17}$ and M. Waldmeier ${ }^{18}$ assuming that no other force than gravitation acts upon the corona, have recently shown that the distribution of electronic density leads to an approximately constant temperature of about $10^{6}$ degrees for the inner corona. The essential result is not altered if the influence of the magnetic fields (general and in sunspots) is introduced. ${ }^{17}$

As mentioned before, the coronal lines have been observed in only two stars, the recurrent novae RS Ophiuchi and $\mathrm{T}$ Pyxidis. Although the $[F e \mathrm{x}]$ line also seems to have been found in two stars AX Persei and CI Cygni, which show extremely strong $[\mathrm{Fe}$ VII] lines, there is no trace of $[F e \mathrm{xIV}]$. Astrophysicists are on the lookout for coronal lines in stellar spectra, because such lines would probably help in the complete understanding of the corona. The $[F e \mathrm{x}]$ line is, of course, the first one to be expected in conditions of excitation lower than those of the solar corona.

The next problem is that of the origin of the high excitation in the corona.

${ }^{16}$ A:N., 263, 121, 1937.

17 Arkiv f. Matem., Astr. och Fysik, 27, A, No. 25, 1941.

18 Zs. f. Ap., 21, 120, 1942. 
We may classify the possibilities of explaining the corona as follows :

a) The production of highly stripped atoms is due to the bombardment of the solar envelope by meteoric matter; in other words, atoms coming from the outside are excited by collision with protons, atoms, or ions present in the corona.

b) The matter and excitation originate in the sun itself.

The meteoric hypothesis is not very satisfactory. A meteoric atom would reach a large velocity of the order of $600 \mathrm{~km} / \mathrm{sec}$ when captured by the sun, and a number of successive collisions with protons, atoms, or ions might strip the atom of several electrons, although the very high observed ionization seems unlikely to be reached in this way. In fact, from the observed line profile, one may conclude that the velocity near the limb does not exceed $60 \mathrm{~km} / \mathrm{sec}$, so that a deceleration of a meteoric atom when it plunges toward the sun is required. One would expect the line widths to decrease with the distance from the limb, which is contrary to the observations. It is also hard to understand why lines, such as $H \alpha$ and other familiar lines, would not be emitted.

Several theories have been advanced to explain the origin of the excitation in the sun. Menzel ${ }^{19}$ suggests that the highly heated coronal matter is emitted in great jets from holes and cracks in the solar surface. "These crevices, whose presence is probably associated with sunspots because the corona is most brilliant in these zones, run far down into the hot interior where the temperature is several million degrees. Coronal jets are the solar analogues of terrestrial volcanoes: gigantic eruptions that send hot material from the interior out to the cooler surface. The gas is somewhat cooled by the expansion as it spreads out into the upper levels. The chief cooling effect, however, appears to be through the radiation of the coronal lines." As far as I know, Menzel's hypothesis has not yet been subjected to detailed mathematical discussion. Lyot's recent observations should have a bearing on such a discussion.

Alfvén has developed another theory according to which the

19 Telescope, 8, 65, 1941. 
supply of high energy particles to the corona (in other words, the heating of the corona) is due to the prominences or, rather, the prominence-like action of the chromosphere. ${ }^{20}$ The total energy necessary to maintain the very high temperature of the corona is small (about $10^{-5}$ of the energy radiated by the sun). Alfvén believes that the motion of solar matter in magnetic fields on the sun, especially the vortical motion in a sunspot, must bring about potential differences between different points of the solar surface. He believes that, under certain conditions, electric discharges will appear and charged particles attain very high energies. It is well known that the coronal material shows a tendency to move in arches or streamers suggestive of magnetic and perhaps also electric fields. Alfvén has studied in considerable detail the effect of the electric and magnetic fields upon the ionized matter of the corona, and has obtained velocities of the $F e$ ions which seem to agree with the line widths observed by Lyot and by Waldmeier. Whatever the final verdict may be on Alfvén's theories, it is probable that the corona, aurora, and magnetic storms are related in some way.

Finally, M. N. Saha ${ }^{21}$ has suggested that the ions of iron and other metals are produced in some kind of nuclear reaction, similar to uranium fission, occurring at some depth below the chromosphere. During its passage through the corona, an $\mathrm{Fe}$ ion would go through successive processes of electron capture or loss, and would eject electrons from the atoms. and ions it encounters.

Saha has published a rather long paper discussing the application of the uranium-fission hypothesis. According to this paper, elements like $\mathrm{Fe}$ and $\mathrm{Ni}$ can be formed only in a threefold or fourfold fission process of the heaviest elements $U r^{239}, U r^{235}$, Prot. $A c^{231}$, or $T h^{232}$, which alone, according to Bohr and Wheeler, are capable of fission. These processes, which have not yet been observed, are possible on energetic grounds.

I am not qualified to discuss the nuclear part of Saha's hy-

20 Many authors regard the chromosphere as a multitude of small prominences.

${ }^{21}$ Proceedings of the National Institute of Sciences of India, 8, 99, 1942. 
pothesis. But, as an astronomer, I am skeptical. The heavy elements are extremely rare in the sun. Moreover, it is hard to see how a continuous supply of neutrons can be found, since neutrons are easily captured by almost all elements. For fission to be important, it is necessary that:

Abundance of the rare isotope of uranium

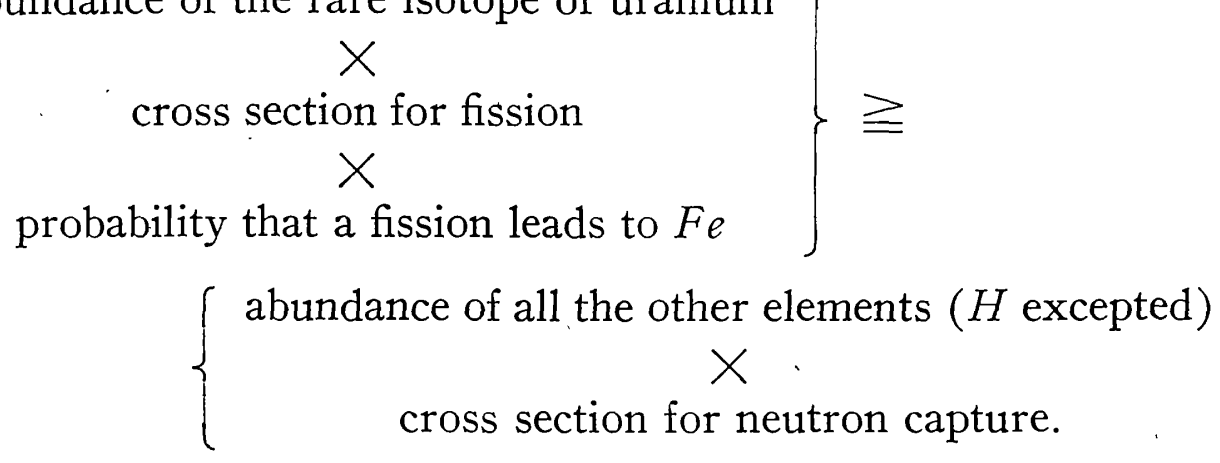

It seems to me doubtful that this can be true.

In peacetime, Eldén's brilliant discovery would certainly have raised many more observational applications and theoretical discussions, and the suggestions of Menzel, Alfvén, and Saha would have been scrutinized in all details. But whatever these discussions may be, the basic point will remain that Edlén brilliantly solved the greatest solar puzzle, just as Bowen solved the greatest spectroscopic enigma concerning the nebulae.

March 1945 